Conclusion Our study confirmed association of DRB1*04 alleles not only with susceptibility to RA but with clinical features of the disease as well.

\section{THU0005 INCREASED FREQUENCY OF HLA DR 11 AND DECREASED FREQUENCY OF HLA DR 1 IN CAUCASIAN EUROPEANS WITH ABDOMINAL AORTIC ANEURYSMS}

${ }^{1} \mathrm{~A}$ Schmarda, ${ }^{1} \mathrm{H}$ Schennach, ${ }^{2} \mathrm{P}$ Klein-Weigel, ${ }^{3} \mathrm{C}$ Goldberger, ${ }^{1} \mathrm{D}$ Schönitzer, ${ }^{4} \mathrm{KP}$ Pfeiffer, ${ }^{2} \mathrm{G}$ Fraedrich, ${ }^{3} \mathrm{M}$ Schirmer. 'Central Institute for Blood Transfusion; ${ }^{2}$ Vascular Surgery; ${ }^{3}$ Internal Medicine; ${ }^{4}$ Biostatistics, Innsbruck University Hospital, Innsbruck, Austria

10.1136/annrheumdis-2001.108

Background A genetic predisposition has been reported to play a key role in the pathogenesis of abdominal aortic aneurysms (AAA) in Japanese and a subgroup of North American patients with inflammatory AAA.

Objectives The aim of this study was to evaluate a possible association of HLA DR phenotypes with AAA in a European population sample.

Methods Consecutive patients with AAA larger than $3 \mathrm{~cm}$ presenting at our outpatient clinic were analysed for HLA class II genotypes using the PCR-SSP technique at low resolution level. DR phenotypes were deduced from these results. Patients with any history of an autoimmune disease were excluded from the study. The frequencies of HLA DR phenotypes were compared to those of a cohort of consecutive and ethnically matched organ donors using the appropriate statistical methods. Classification trees have been applied to increase the predictive value and to consider more than one variable.

Results HLA DR genotyping was performed in 54 European AAA patients (44 males) and 296 controls (203 males). At the HLA DRB1* locus, 19/54 AAA patients $(35,2 \%)$ and 63/296 controls $(21,3 \%)$ were found to be $1 * 11$ positive $(\mathrm{p}=0,035$; $\mathrm{OR}=2,01,95 \%$ c.i.: $1,08-3,75)$. Subanalysis of male patients revealed 17/44 (38,6\%) AAA patients and 39/203 (19,2\%) in controls to be $1 * 11$ positive ( $\mathrm{p}=0,009$; $\mathrm{OR}=2,65,95 \%$ c.i.: 1,31-5,33). Regarding HLA DRB1*01, 4/54 AAA patients $(7,4 \%)$ and $64 / 296$ controls $(21,6 \%)$ turned out to be $1 * 01$ carrier $(\mathrm{p}=0,014$; OR $=0,29,95 \%$ c.i.: $0,10-0,83)$. Subanalysis of male patients revealed $2 / 44(4,5 \%)$ and 48/203 (23,6\%) DRB1 $* 01$ carrier in AAA patients and controls, respectively $(\mathrm{p}$ $=0,003 ;$ OR $=0,15,95 \%$ c.i.: $0,04-0,66)$. No associations for DRB $1 * 11$ and $1 * 01$ were observed in female patients.

Conclusion These data suggest a positive association between AAA and DR 11 and a possible protective effect of DR 1 in male Caucasian Europeans suffering from AAA. This finding supports the hypothesis that MHC class II genes play a role in the pathogenesis of AAA.

\section{THU0006 DQB1 POLYMORPHISM IN RHEUMATOID ARTHRITIS PATIENTS WITH SHARED EPITOPE-NEGATIVE DRB1 ALLELES}

${ }^{1} \mathrm{R}$ Zunec, ${ }^{1} Z$ Grubic, ${ }^{2} \mathrm{D}$ Babic-Naglic, ${ }^{2} \mathrm{~N}$ Laktasic-Zerjavic, ${ }^{3} \mathrm{~K}$ Potocki, ${ }^{1} \mathrm{~A}$ Kastelan. ${ }^{1}$ Tissue Typing Center; ${ }^{2}$ Clinic of Rheumatology; ${ }^{3}$ Clinic of Radiology, University Hospital Zagreb, Zagreb, Croatia

\subsection{6/annrheumdis-2001.109}

Background The association of rheumatoid arthritis (RA) with HLA-DRB1 alleles sharing sequences in the third hypervariable region has been reported in several investigations, but genetic analyses suggest the possible involvement of other HLA class II genes in conferring susceptibility.

Objectives We have investigated the distribution of HLA-DQB1DRB1 polymorphism in 87 RA patients from southern Croatia and 141 healthy controls. RA patients were predominantly females (83 females vs 5 males).

Methods All patients and controls were tested for HLA class II alleles (DRB1, DQA1, and DQB1) by PCR-SSP method.

Results Distribution of predisposing DRB1 alleles according to the shared epitope (SE) model showed that 11 patients were homozygous for the predisposing alleles. Additional 48 patients carried one of the SE predisposing alleles $(* 0101, * 1001, * 0401$, $* 0404, * 0405, * 0408)$. Distribution of DQB1 alleles among patients with SE-positive DRB1 alleles revealed that all DRB1*0101 and *1001 patients were DQB1*0501 positive. Among twenty patients with SE-positive DRB1*04 alleles, three different DQB1 alleles were observed (DQB1*0301, "0302 and *0201). Remaining 26 patients with SE-negative DRB1 alleles were additionally analysed for the presence of DQB1*0301, *0302 and *0201 alleles. All eight patients with SE negative DRB1*04 alleles (DRB1*0402, *0403, *0407) were DQB1*0302 positive. Among the remaining non DRB1*04 patients, 16 were either DQB1*0301 or *0201 and only two patients did not carry neither DQB1*0301 nor *0201.

Conclusion These data suggest that in patients with SE negative DRB1 alleles, DQB1*0301, *0302 and *0201 alleles should be considered as a genes contributing to the RA predisposition. DQB1*0301, "0302 and *0201 alleles should be considered as a genes contributing to the RA predisposition.

\section{THU0007 ASSOCIATION OF HUMAN MHC CLASS I AND CLASS II GENES WITH SUSCEPTIBILITY TO SYSTEMIC LUPUS ERYTHEMATOSUS IN MALAYS AND CHINESE}

${ }^{1} \mathrm{SS}$ Yeap, ${ }^{2} \mathrm{KF}$ Kong, ${ }^{2} \mathrm{YP}$ Tee, ${ }^{1} \mathrm{SK}$ Chow, ${ }^{2} \mathrm{ME}$ Phipps. 'Department of Medicine; ${ }^{2}$ Department of Allied Health Sciences, University of Malaya, Kuala Lumpur, Malaysia

\subsection{6/annrheumdis-2001.110}

Background Systemic lupus erythematosus (SLE) is a chronic autoimmune disorder. Its prevalence is higher in Chinese and Black populations compared to Caucasians. The prevalence of SLE in Malaysia is estimated to be $43 / 100,000$ population. ${ }^{1}$

Objectives The objective of this study was to investigate the association between SLE with the human MHC Class I and Class II genes.

Methods A modified PCR-SSP Methodology, known as Phototyping $^{2,3}$ was carried out to determine the HLA-A, -B, -DR and DQ genes of the subjects. 50 Malays and 50 Chinese patients with SLE fulfilling the ACR criteria were recruited along with 50 Malays and Chinese age- and race-matched controls.

Results Statistical analysis showed that both the Malay and Chinese have similar results despite minor differences. HLA-A11 is associated with SLE in both races, whereas HLA-DR12 has been found to be statistically significant as protective antigen specificity. Compared to Malays, Chinese SLE patients have a higher frequency of DR2 compared to the controls. DRB1 was only seen in Malay SLE patients (OR 8.69 compared to controls). DRB1*02 and DQB1*05 was only seen in Chinese SLE patients (OR 2.41 compared to controls). Alleles negatively associated with SLE, possibly confering a protective effect are as follows: In both Malay and Chinese SLE patients, DRB1*12, 